\title{
Inert gas accumulation in sonoluminescing bubbles
}

\author{
Detlef Lohse ${ }^{\text {a) }}$ and Sascha Hilgenfeldt ${ }^{\text {b) }}$ \\ Fachbereich Physik der Universität Marburg, Renthof 6, 35032 Marburg, Germany
}

(Received 31 March 1997; accepted 25 July 1997)

\begin{abstract}
In this paper we elaborate on the idea [Lohse et al., Phys. Rev. Lett. 78, 1359-1362 (1997)] that (single) sonoluminescing air bubbles rectify argon. The reason for the rectification is that nitrogen and oxygen dissociate and their reaction products dissolve in water. We give further experimental and theoretical evidence and extend the theory to other gas mixtures. We show that in the absence of chemical reactions (e.g., for inert gas mixtures) gas accumulation in strongly acoustically driven bubbles can also occur. (C) 1997 American Institute of Physics. [S0021-9606(97)51241-4]
\end{abstract}

\section{INTRODUCTION}

Sonoluminescence (SL) has long been known to be very sensitive to the gas used. ${ }^{1-5}$ This effect is even more pronounced for single bubble sonoluminescence (SBSL), a phenomenon in which a single gas bubble is driven by a strong acoustic field and can emit short light pulses for hours. ${ }^{6,7}$ Detailed experiments by the Putterman group at UCLA ${ }^{8-16}$ revealed that inert gas as part of the operating gas is essential. In Figure 1 we show the experimental SL intensity from a SL bubble in water as a function of the percentage $\xi_{l}$ of noble gas mixed with nitrogen, taken from Ref. 11. Pure nitrogen bubbles show hardly any SL emission; the optimum is around $\xi_{l}=0.01=1 \%$, i.e., the amount of argon contained in air.

Besides the noble gas percentage $\xi_{l}$ the other experimentally controllable parameters in SBSL experiments are the forcing pressure amplitude $P_{a}$ of the forcing sound field

$$
P(t)=P_{a} \cos \omega t,
$$

the total gas pressure overhead $p_{\infty}$, and the ambient pressure $P_{0}$, which is normally $1 \mathrm{~atm}$. The frequency $\omega / 2 \pi$ which is in a range between $20 \mathrm{kHz}$ and $40 \mathrm{kHz}$ is fixed as it has to be adopted to the size of the resonator ("Crum cell," ${ }^{17}$ ). Note that the ambient radius $R_{0}$ of the bubble (i.e., the bubble radius at ambient standard conditions $P_{0}=1 \mathrm{~atm}$ and at the temperature of the water) is not a free parameter, but the system adjusts $R_{0}$ itself by diffusional processes.

There are two types of SBSL: stable and unstable SBSL. ${ }^{13,18}$ In unstable SBSL the phase of light emission (measured relative to the phase of the forcing sound field) drifts on a diffusional time scale of seconds, interrupted by sudden breakdowns. The same is true for the maximal bubble size per cycle and for the light intensity. An example for the bubble dynamics and light intensity for unstable SBSL is shown in Figure 6 of Ref. 15. In Ref. 18 we quantitatively accounted for unstable SBSL as light emission from a bubble growing by rectified diffusion ${ }^{5,15,18-22}$ and thereby running into a shape instability ${ }^{5,18,22-25}$ where it pinches off a microbubble and starts over. The pinch off leads to a recoil of the bubble which makes it appear "jiggling" or "dancing", 6,7,13 Meanwhile, the pinched off mi-

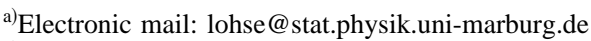

b) Electronic mail: hilgenfeldt@ @stat.physik.uni-marburg.de
}

crobubble could be visualized. ${ }^{26}$ The second type of SBSL is stable SBSL, distinguished by a constant phase and intensity of the light pulses, repeating for hours with remarkable precision. $8,9,27$

In Ref. 18 we calculated phase diagrams of SL bubbles in the ambient radius vs forcing pressure and gas pressure vs forcing pressure phase spaces. These diagrams are based on the Rayleigh-Plesset equation for the bubble radius $R(t)$, a similar type of approximation for shape distortions, and the advection diffusion equation. We call this approach the Rayleigh-Plesset SL bubble approach. Shape stabilities and diffusional stabilities are considered. The phase diagrams quantitatively agree with Barber et al.' ${ }^{13}$ and Löfstedt et al.' $\mathrm{s}^{15}$ experiments for argon (and other inert gas) bubbles, but $n o t$ for air bubbles.

For argon bubbles stable SBSL is only possible in a small window of tiny gas concentration, see Figure 2 which is calculated for a water temperature of $20^{\circ} \mathrm{C}$, as all calculations done in this paper. For $P_{a}=1.3 \mathrm{~atm}$ this window is between $p_{\infty}^{\mathrm{Ar}} / P_{0}=0.002$ and 0.004 in very good agreement with Löfstedt et al.'s experimental data. Repeating the calculation of such phase diagrams for air bubbles essentially gives the same result, however, experimentally stable SBSL in air bubbles is found at about one hundred times larger gas pressure overhead. ${ }^{6,13,22}$ This discrepancy between air and argon bubbles was first pointed out by Löfstedt et al. ${ }^{15}$ who hypothesized an "as yet unidentified mass ejection mechanism" in air bubbles which "is the key to SL in a single bubble."

\section{AIR BUBBLES VS ARGON BUBBLES}

In Refs. 28 and 29 we have suggested that this mechanism is chemical. The occurrence of chemical reactions has in fact led to the discovery of multibubble sonoluminescence $(\mathrm{MBSL})^{2-5,30-32}$ : Frenzel and Schultes ${ }^{1}$ were stimulated to look for luminescence as the formation of hydrogen peroxide in aqueous fluids subjected to sound that had been observed before. Later, Schultes and Gohr ${ }^{33}$ found that also nitric and nitrous acids were produced. The reason is that the high temperatures generated by the bubble collapse are beyond the dissociation temperature of oxygen and nitrogen $\left(\approx 9000 \mathrm{~K}^{34}\right)$, leading to the formation of $\mathrm{O}$ and $\mathrm{N}$ radicals which react with the $\mathrm{H}$ and $\mathrm{O}$ radicals formed from the dissociation of water vapor. Rearrangement of the radicals will 


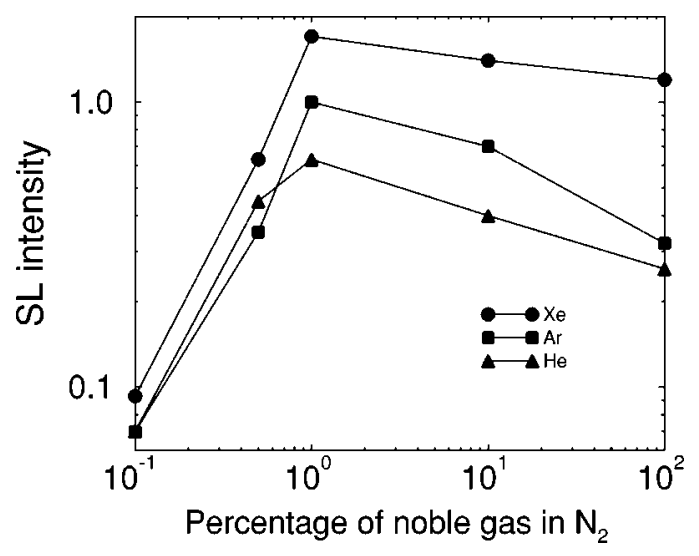

FIG. 1. SL intensity (normalized to air) from a SL bubble in water as a function of the percentage (mole fraction) of noble gas mixed with nitrogen. The gas mixture was dissolved in water at a pressure head of $150 \mathrm{~mm} \mathrm{Hg}$, i.e., $p_{\infty} / P_{0} \sim 0.2$. The data are taken from Figure 1 of Hiller et al. (Ref. 11).

lead to the formation of $\mathrm{NO}, \mathrm{OH}$, and $\mathrm{NH}$, which eventually dissolve in water to form $\mathrm{H}_{2} \mathrm{O}_{2}, \mathrm{HNO}_{2}$, and $\mathrm{HNO}_{3}$, among other products.

Based on fits of SBSL spectra ${ }^{10,35}$ and hydrodynamic calculations, ${ }^{14,36}$ it is assumed that internal bubble temperatures in SBSL are even higher than in MBSL. Therefore, the same reactions as in MBSL will occur. The reaction products $\left(\mathrm{NO}_{2}, \mathrm{NO}, \ldots\right)$ are pressed into the surrounding liquid, and are not recollected during the next bubble cycle, since their solubility in water is enormous. These chemical processes deprive the gas in the bubble of its reactive components. Small amounts of $\mathrm{N}_{2}$ and $\mathrm{O}_{2}$ that diffuse into the bubble during the expansion react and their dissociation products are expelled back into the surrounding liquid at the bubble collapse. The only gases that can remain within a SBSL bubble over many bubble cycles are those which at high temperatures do not react with the liquid vapor, i.e., inert gases. Hence, when air is dissolved in water, a strongly forced bubble is almost com-

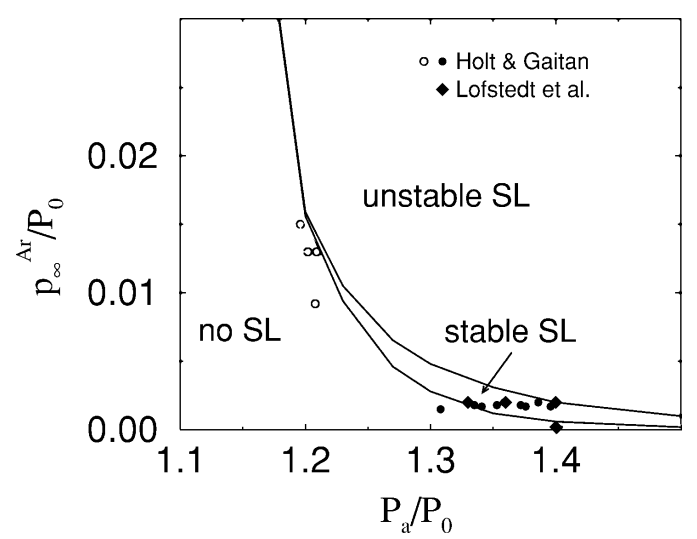

FIG. 2. Phase diagram for pure argon bubbles in the $p_{\infty}^{\mathrm{Ar}} / P_{0}$ versus $P_{a} / P_{0}$ parameter space. Stable SL is only possible in a very small window of argon concentration. The experimental data points included for comparison refer to observed stable SL (filled symbols) or stable non-SL bubbles (open symbols) from Refs. 15 (diamonds) and 22 (circles) and show good agreement with the theory. Note that only those data can be included for which $P_{a}, p_{\infty}$ and $\xi_{l}$ are experimentally known. pletely filled with argon. This argon rectification happens in SBSL but not in MBSL because it requires long time bubble stability.

This argument immediately suggests that the partial pressure of argon

$$
p_{\infty}^{A r}=\xi_{l} p_{\infty}
$$

determines bubble stability, not the total pressure $p_{\infty}$. Indeed, if we include Holt and Gaitan's experimental data for air bubbles ${ }^{22}$ on inert gas-nitrogen mixtures and only consider the inert gas partial pressure as the relevant quantity for diffusive stability, excellent agreement with the theoretical phase diagrams ${ }^{18}$ is found.

The phase diagram shows that for $P_{a}=1.3$ atm argon bubbles exist between $0.002<p_{\infty}^{\mathrm{Ar}} / P_{0}<0.004$. For pure argon dissolved in water, $p_{\infty}^{\mathrm{Ar}}=p_{\infty}$. For air bubbles, however, the partial pressure of argon is only $p_{\infty}^{A r}=0.01 p_{\infty}$ which requires $0.2<p_{\infty} / P_{0}<0.4$ for stable SL with " air' bubbles, in good agreement with experiment. Since this amount of degassing is easily achieved, air with its $1 \%$ argon is a particularly friendly gas for SL experiments. The theory suggests that for an argon ratio of $\xi_{l} \approx 0.0033$ at $P_{a}=1.3 \mathrm{~atm}$ there is stable SL between $0.6<p_{\infty} / P_{0}<1.2$, so that degassing is not required. Also, the window of stability is even wider than for air. The major problem in experimentally achieving SBSL without degassing is spontaneous cavitation, provoked by impurities in the liquid. These must be eliminated for the experiment to work. Another way to obtain stable SL without degassing would be to slightly increase the ambient pressure $P_{0}$ so that the ratio $p_{\infty}^{\mathrm{Ar}} / P_{0}$ is in the required window. Indeed, with such a kind of experiment stable SBSL without degassing could recently be achieved. ${ }^{37}$

Before we proceed with a quantitative analysis, we will give further support for the nitrogen dissociation hypothesis ${ }^{28,29}$ from comparison with various experimental results on gas mixtures.

(1) Transition toward SL: The transition toward SL with increasing forcing pressure $P_{a}$ is shown in Figure 3 for both argon and air bubbles. For pure argon bubbles the transition to SL is very smooth. For air, however, one can observe a breakdown of the bubble radius at about 1.1 atm, signaling that the dissociation threshold of $\mathrm{N}_{2}$ is achieved. Before the transition the bubble is filled with a mixture of nitrogen, oxygen, and argon, and the ambient radius is determined by the combination of all three gases. Beyond the dissociation threshold, only argon is left in the bubble. The transition from the no SL regime in Figure 2 to the SL regime can also be seen in Figure 1, where the SL light intensity is plotted as a function of $\xi_{l}$ for fixed $P_{a}$ (we assume $\left.P_{a}=1.3 \mathrm{~atm}\right)$ and fixed $p_{\infty} / P_{0} \sim 0.20$. According to our theory we expect SL for $p_{\infty}^{\mathrm{Ar}} / P_{0}>0.002$ or

$$
\xi_{l}>\frac{p_{\infty}^{A r} / P_{0}}{p_{\infty} / P_{0}}=0.01,
$$

in pretty good agreement with Figure 1 where we indeed observe that strong SL is "switched on" at about that concentration. Our theory also predicts that near the switch on we always have stable SL, whereas for larger $\xi_{l}$ unstable SL 


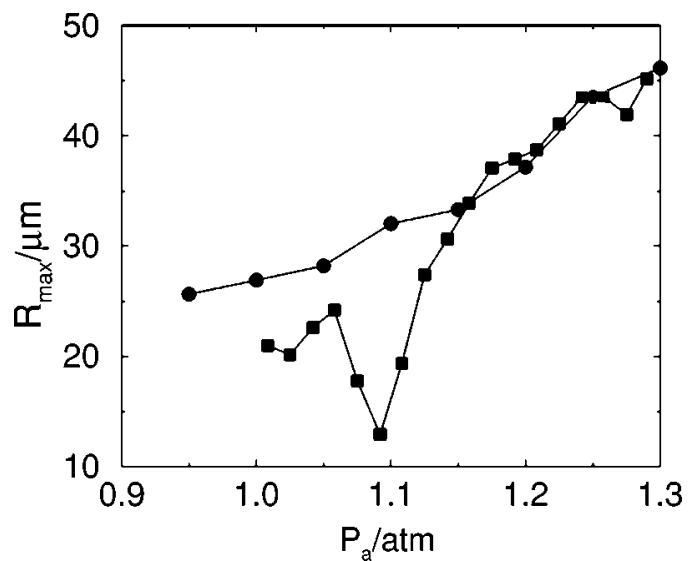

FIG. 3. Transition in the maximal radius towards the SL regime for argon bubbles (dots) and for air bubbles (squares). Bubbles around $P_{a}=1.1 \mathrm{~atm}$ start to glow. Only for air bubbles a breakdown in the radius is seen near that onset of SL, signaling the threshold for nitrogen dissociation. The data for argon are taken from Figure 4 of Hiller et al.'s work (Ref. 11), the total gas concentration is about $p_{\infty}=150 \mathrm{~mm} \mathrm{Hg}$. This means $p_{\infty}^{\mathrm{Ar}} / P_{0}=0.2$ and according to Figure 2 the bubble should be in the unstable SL regime in agreement with the observations of Ref. 11. The data for air are taken from Figure 2 of Barber et al.'s work (Ref. 36). The gas saturation is about 10\%, corresponding to $p_{\infty}^{\mathrm{Ar}} / P_{0}=0.01 \cdot 0.1=0.001$. According to Figure 2 we have stable SL around $P_{a} \sim 1.4$ atm which again is in agreement with the experiment reported in Ref. 36.

develops. The window of stable SL is only in between $0.002<p_{\infty}^{\mathrm{Ar}} / P_{0}<0.004$, so that beyond $\xi_{l}=0.004 P_{0} / p_{\infty}$ we expect unstable SL, a prediction which should be verified.

(2) Hysteresis: Above we have seen that by decreasing the percentage $\xi_{l}$ of inert gas down to $\xi_{l} \approx 0.0033$ at $P_{a}=1.3 \mathrm{~atm}$, we obtain a very wide window of stable SL around $p_{\infty} / P_{0}=1$. When the percentage $\xi_{l}$ of inert gas is even lower, the argon partial pressure $p_{\infty}^{\mathrm{Ar}} / P_{0}=\xi_{l} p_{\infty} / P_{0}$ can be pushed below the stable SL regime in Figure 2 even for $p_{\infty} / P_{0}=1$. An example of this has been observed in Figure 11 of Löfstedt et al. ${ }^{15}$ see Figure 4 of the present work, which studies SBSL of xenon doped nitrogen bubbles (which behave like argon doped nitrogen bubbles) in water at $\xi_{l}=0.001$ and $p_{\infty} / P_{0}=0.2$, corresponding to $p_{\infty}^{\mathrm{Ar}} / P_{0}$ $=0.0002$, provided that the gas temperature is high enough to exceed the dissociation temperature of nitrogen. This is the case at $P_{a}=1.3 \mathrm{~atm}$ and according to Figure 2 no stable $\mathrm{SL}$ is possible. However, for larger $P_{a}=1.4 \mathrm{~atm}$ stable SL becomes possible again (as the window of stable SL moves down to smaller concentrations with increasing $P_{a}$ ), just as observed in experiment. Moreover, the system shows hysteresis: the SL state at $P_{a}=1.4 \mathrm{~atm}$ can be reached by continuously increasing the forcing and thus continuously substituting $\mathrm{N}_{2}$ with xenon. On the other hand, decreasing the forcing pressure below the stability threshold for pure xenon bubbles leads to the dissolution of the bubble.

(3) Unstable SL: An example for unstable SL is shown in Figure 6 of Ref. 15. For that figure we have $\xi_{l}=0.05$ and $p_{\infty} / P_{0}=0.20$. Thus, from Equation (2) we have $p_{\infty}^{\mathrm{Ar}} / P_{0}=0.01$ and according to the phase diagram Figure 2 we are well in the unstable SL regime, just in agreement with the observations.

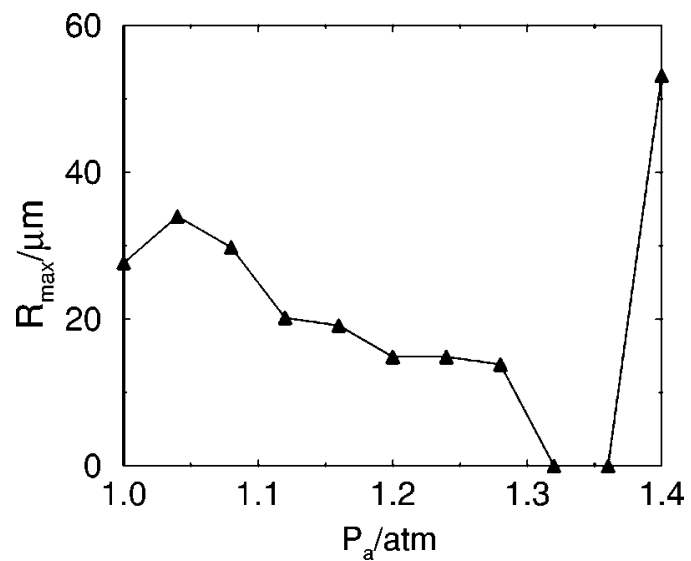

FIG. 4. The transition (in $R_{\max }$ ) to SL for a bubble filled with an initial $0.1 \%$ xenon in nitrogen gas mixture at a partial pressure of $150 \mathrm{~mm} \mathrm{Hg}$. Only the bubble at $P_{a}=1.4$ atm emits light. According to the dissociation hypothesis this corresponds to $p_{\infty}^{\mathrm{Xe}} / P_{0}=0.0002$ for a pure sonoluminescing xenon bubble. From the phase diagram Figure 2 which is (with tiny corrections) also valid for xenon we conclude that bubbles driven at $P_{a}=1.3 \mathrm{~atm}$ dissolve for these low concentrations, whereas bubbles at $P_{a}=1.4$ atm show stable SL, just as seen here. The data are taken from Figure 11 of Löfstedt et al. (Ref. 15).

(4) Large $P_{a}$ bubble dissolution islands: Further support comes from the recent work of Holt and Gaitan, ${ }^{22}$ who measured detailed phase diagrams as a function of the ambient radius $R_{0}$ and the forcing pressure $P_{a}$ for different air pressure overhead $p_{\infty} / P_{0}$. Their central result is that at $p_{\infty} / P_{0}=0.2$ there is a relatively large forcing pressure $P_{a} \sim 1.2-1.3 \mathrm{~atm}$ regime where bubbles dissolve, see Figure 5. Such dissolution islands do not exist within theories of

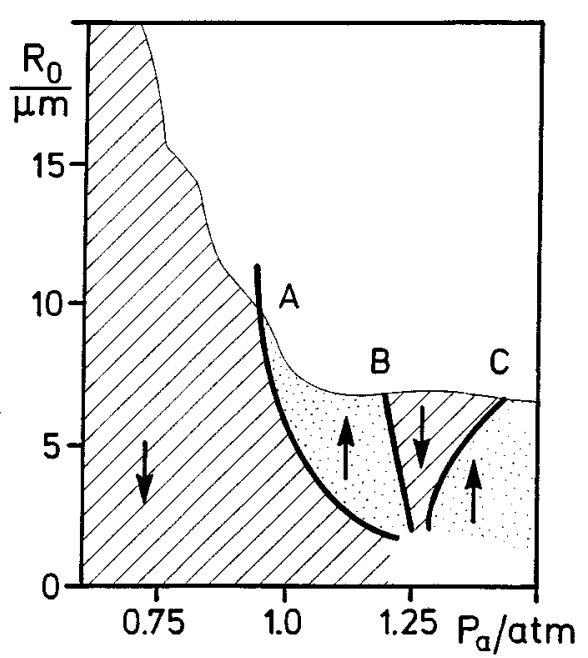

FIG. 5. Adopted from Holt and Gaitan's measurement Figure 1(c) of Ref. 22. It is the phase diagram in the $R_{0}-P_{a}$ parameter space for air at $p_{\infty} / P_{0}=0.20$; the driving frequency is $20.6 \mathrm{kHz}$. Arrows indicate whether the bubbles grow or shrink. Three equilibrium curves $\mathrm{A}, \mathrm{B}$, and $\mathrm{C}$ can be recognized. In between curves B and C there is a "dissolution island." The shaded area shows the shape stable parameter domain. Note that in the SL region the onset of shape instabilities is at $R_{0} \approx 7 \mu \mathrm{m}$ rather than at $R_{0} \approx 5 \mu \mathrm{m}$ as theoretically calculated in Ref. 18 . One contribution to this deviation is that the driving frequency chosen by Holt and Gaitan is smaller than the $\omega / 2 \pi=26.5 \mathrm{kHz}$ used by Barber et al. (Ref. 36) and throughout the calculations presented here; another one is the oversimplified model of thermal effects we use. 
rectified diffusion, ${ }^{15,18-20}$ which predict the bubbles to grow in that regime. However, considering the nitrogen dissociation at large $P_{a}$, one realizes that the observed dissolution islands are a direct consequence: chemical reactions deplete the bubbles from air and only argon is left. As the relevant partial pressure $p_{\infty}^{\mathrm{Ar}} / P_{0}=0.002$ is so low, bubbles must indeed shrink in that regime.

(5) Isotope scrambling: Experiments with hydrogen gas also support the dissociation hypothesis. Hiller et al. ${ }^{12}$ analyze SL in $\mathrm{H}_{2}$ and $\mathrm{D}_{2}$ gas bubbles, both in normal and in heavy water. Since the gas dynamics inside the bubble determines the strength of the light emission, the SL intensity curves should group according to the gas content. However the four experiments $\left(\mathrm{H}_{2}\right.$ in $\mathrm{H}_{2} \mathrm{O}, \mathrm{H}_{2}$ in $\mathrm{D}_{2} \mathrm{O}, \mathrm{D}_{2}$ in $\mathrm{H}_{2} \mathrm{O}$, and $\mathrm{D}_{2}$ in $\mathrm{D}_{2} \mathrm{O}$ ) group according to the surrounding liquids, cf. Figure 2 of Ref. 12. This suggests the following scenario: Both the gas and the liquid vapor in the bubble dissociate to some extent during the (hot) compression phase and recombine later on during expansion. Even if there is only a minor amount of this isotope "scrambling," after thousands of cycles the gas in solution around the bubble would contain the same hydrogen isotope as the bulk liquid. This kind of "scrambling", is well known in MBSL. ${ }^{38}$ The acoustic resonator theory of SBSL $^{39}$ stipulates that the light intensity decreases with increasing acoustic transmission, which scales with the ratio of the gas density to the liquid density. Since $\mathrm{D}_{2}$ is heavier than $\mathrm{H}_{2}$, SBSL in heavy water should be dimmer than in normal water, as observed by Hiller and Putterman. ${ }^{12}$

\section{MODELING THERMAL EFFECTS WITHIN RAYLEIGH-PLESSET BUBBLE DYNAMICS}

We now proceed to a quantitative calculation of phase diagrams for gas mixtures. The dynamics of the bubble radius $R(t)$ is well described by the Rayleigh-Plesset equation, ${ }^{5,40}$

$$
\begin{aligned}
R \ddot{R}+\frac{3}{2} \dot{R}^{2}= & \frac{1}{\rho_{l}}\left(p(R, t)-P(t)-P_{0}\right)+\frac{R}{\rho_{l} c_{l}} \frac{d}{d t} p(R, t) \\
& -4 \nu \frac{\dot{R}}{R}-\frac{2 \sigma}{\rho_{l} R}
\end{aligned}
$$

with a van der Waals pressure

$$
\gamma\left(R_{1} t\right)=p(R(t))=\left(P_{0}+\frac{2 \sigma}{R_{0}}\right)\left(\frac{R_{0}^{3}-h^{3}}{R^{3}(t)-h^{3}}\right)^{\gamma} .
$$

Typical parameters for an argon bubble in water at room temperature $^{13}$ are the surface tension $\sigma=0.073 \mathrm{~kg} / \mathrm{s}^{2}$, the water viscosity $\nu=10^{-6} \mathrm{~m}^{2} / \mathrm{s}$, density $\rho_{l}=1000 \mathrm{~kg} / \mathrm{m}^{3}$, the polytropic exponent $\gamma=1$, and speed of sound in water $c_{l}=1481 \mathrm{~m} / \mathrm{s}$. For all calculations in this work we picked the same driving frequency as in the SL experiments performed on argon bubbles, ${ }^{13}$ namely $\omega / 2 \pi=26.5 \mathrm{kHz}$, corresponding to a period $T=2 \pi / \omega=38 \mu \mathrm{s}$. Finally, $h=R_{0} / 8.86$ is the hard core van der Waals radius for argon bubbles. ${ }^{14}$ Typical time series for the bubble radius $R(t)$ resulting from Equation (4) for given forcing $P(t)$ are shown in Figure 6.

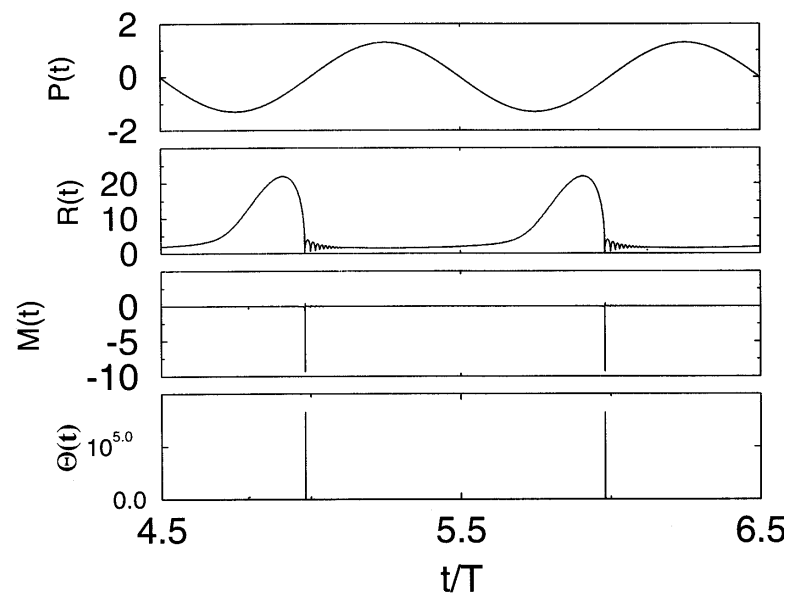

FIG. 6. Forcing pressure (in atm) with amplitude $P_{a}=1.3 \mathrm{~atm}$, bubble radius $R(t)$ (in $\mu \mathrm{m}$ ) with the typical collapse ("Rayleigh collapse") after the maximum, Mach number $M(t)=\dot{R}(t) / c_{g}(t)$ (where $c_{g}$ is the speed of sound in the gas), and temperature $\Theta(t)$ (in $\mathrm{K}$ ) as function of time for a bubble with $R_{0}=2 \mu \mathrm{m}$ (top to bottom).

Thermal conduction effects can approximately be taken into account by simply putting the polytropic exponent $\gamma=1$ in Equation (5). The reason is that the bubbles employed in SL experiments are so tiny and the oscillation period $T \approx 38 \mu$ s so long that the gas in the bubble equilibrates with the water temperature $\Theta_{l}$. A quantitative analysis was carried out by Prosperetti ${ }^{41}$ (see also the review in Ref. 42) who calculated how the polytropic exponent $\gamma$ depends (in linear approximation) on the (thermal) Peclet number $P e=R_{0}^{2} \omega / \kappa$, see Figure 1 of Ref. 42. For experimental support of this analysis, see Crum. ${ }^{43}$ The Peclet number gives the ratio between the bubble length scale $R_{0}$ and the thermal diffusion length $\sqrt{\kappa / \omega}$. The thermal diffusivity $\kappa$ for argon is $\kappa \approx 2 \times 10^{-5} \mathrm{~m}^{2} / \mathrm{s}$, which yields $P e \approx 0.2$ for $R_{0}=5 \mu \mathrm{m}$ and according to Figure 1 of Ref. 42, the polytropic exponent $\gamma=1$.

The RP equation obviously contains much smaller time scales than $\omega^{-1}$. One could therefore argue that these smaller time scales may enter into the calculation of $P e$, so that the frequency $\omega$ should be replaced by $|\dot{R}| / R$. This estimate leads to an instantaneous Peclet number

$$
P e=\frac{|\dot{R}| R_{0}^{2}}{R \kappa},
$$

which can become as large as $10^{4}$ at the Rayleigh collapse. According to Figure 1 of Ref. 42 showing $\gamma(\mathrm{Pe})$ or Prosperetti, Crum, and Commander's fit of that curve, ${ }^{44}$ implies $\gamma \approx 5 / 3$ for argon at the time of the collapse. However, since $P e(t) \gg 1$ only holds in very small time intervals $\sim 1 \mathrm{~ns}$, the global dynamics are hardly affected by setting the effective polytropic exponent $\gamma=1$ uniformly in time. Note that with $\gamma=1$ Equation (5) should not be thought of as an equation of state but rather as a process equation parametrizing the isothermal conditions at the bubble wall, induced by the large heat capacity of water. The choice of $\gamma=1$ is confirmed by the full numerical simulations of Vuong and Szeri ${ }^{45}$ and by 
the analysis of Kamath et al. ${ }^{46}$ Note that, as a consequence, there are heat fluxes back and forth across the bubble wall.

Another confirmation for putting $\gamma=1$ for the bubble dynamics comes from a recent work of Yasui, ${ }^{47}$ who modeled the heat flow through the bubble wall and also included water evaporation and condensation. Consideration of these effects ${ }^{47}$ fits the experimentally measured $R(t)$ curves ${ }^{13,14,36}$ pretty well. ${ }^{48}$ However, the essence of the dynamics is the same as with simply putting $\gamma=1$ and the phase diagrams we are going to calculate in this paper are hardly affected by neglecting the details of the thermal effects.

Even if thermal effects are simply treated by putting $\gamma=1$ for most of the time during the bubble oscillation, they are essential at the final stage of the collapse and thus for estimates of the temperature achieved in the bubble. The present understanding ${ }^{5,44}$ is that the only way to reliably predict the temperatures in the bubble is a full numerical simulation of the gas dynamical equations inside the bubble. Clearly, for strong enough forcing, shock waves will bounce back and forth inside the bubble and the spatial temperature distribution in the bubble will be highly inhomogeneous. ${ }^{49}$

Such full numerical calculation is not within the spirit of the Rayleigh-Plesset SL bubble approach. ${ }^{18}$ We therefore come back to Prosperetti's ${ }^{41}$ model and estimate also in the case of nonlinear bubble oscillations the temperature within that model. For example, we calculate the instantaneous Peclet number (6), determine the resulting effective polytropic exponent $\gamma_{\text {eff }}$ from Figure 1 of Ref. 42 and assume a van der Waals behavior of the internal gas according to

$$
\Theta(t)=\Theta_{l}\left(\frac{R_{0}^{3}-h^{3}}{R^{3}(t)-h^{3}}\right)^{\gamma_{\mathrm{eff}}-1},
$$

where $\Theta_{l}$ is the temperature of the liquid. This, as any model which neglects spatial inhomogeneities in the gas, is a very crude model for the temperature and will only be able to give orders of magnitude. The physical statements resulting from it, however, do not depend on details of this model and so we feel that such an approach is justified.

It can be seen from Figures 7c and 6d that this temperature model gives the correct trends. In Figure $7 \mathrm{c}$ we show the maximal $\gamma_{\text {eff }}$ per cycle as a function of $P_{a}$. We also give the minimal and the maximal bubble radius in Figures $7 \mathrm{a}$ and $\mathrm{b}$. For small $P_{a}$ the maximal effective polytropic exponent $\gamma_{\text {eff }}$ is close to one and jumps toward $5 / 3$ at the transition from the sinusoidal to the bouncing bubble $R(t)$ dynamics. This transition has been analyzed in detail in Ref. 50. The temperature $\Theta(t)$ from Equation (7) is shown in Figure 6d. It strongly peaks at the collapse; for all other times it essentially equals the liquid temperature $\Theta_{l}(=293 \mathrm{~K}$ in this example). The heating at the collapse can be enormous.

What are the temperatures achieved in this approach? In Figures $7 \mathrm{~d}$ and 8 we plot the maximal temperature within our model as a function of $R_{0}$ and $P_{a}$. In the parameter regime of interest for SL, values as high as 20000-60000 K are achieved. These values agree order of magnitude-wise with the independent results of Bernstein et al. ${ }^{35,51}$ who extracted
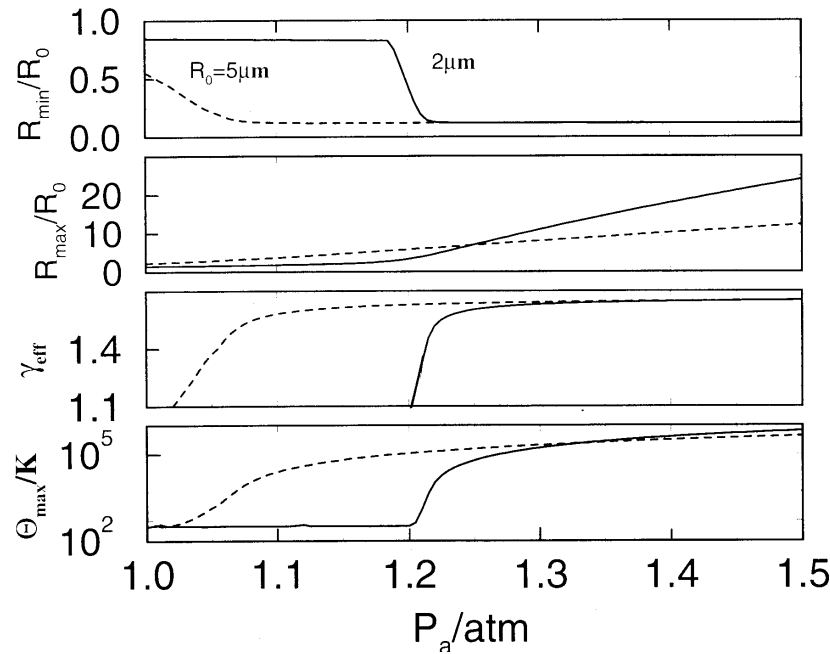

FIG. 7. The minimal radius, the maximal radius, the maximal effective polytropic exponent $\gamma_{\text {eff }}$, and the maximal temperature $\Theta_{\max }$ are shown for two different ambient radii $R_{0}=2 \mu \mathrm{m}$ (solid) and $R_{0}=5 \mu \mathrm{m}$ (dashed) as a function of the forcing pressure amplitude $P_{a}$. For smaller bubbles the crossover from the harmonically oscillating regime for small $P_{a}$ to the bouncing bubble regime for large $P_{a}$ is more abrupt

the temperature from the spectral shape and obtained 30000 to $60000 \mathrm{~K}$, depending on the gas contents.

\section{PHASE DIAGRAMS FOR AIR BUBBLES}

Now consider a bubble in water containing a mixture of a reactive gas (taken to be $\mathrm{N}_{2}$ ) and an inert gas Ar. The total number of moles of gas in the bubble is

$$
N_{t o t}=\frac{4 \pi R_{0}^{3} P_{0}}{3 G \Theta_{0}}=N_{\mathrm{N}_{2}}+N_{\mathrm{Ar}},
$$

where $\Theta_{0}=273 \mathrm{~K}$ is the normal temperature and $G=8.3143 \mathrm{~J} /(\mathrm{mol} \mathrm{K})$ is the gas constant. The argon ratio in the bubble is

$$
\xi_{b}=\frac{N_{\mathrm{Ar}}}{N_{\text {tot }}},
$$

and that of nitrogen

$$
1-\xi_{b}=\frac{N_{\mathrm{N}_{2}}}{N_{t o t}} \text {. }
$$

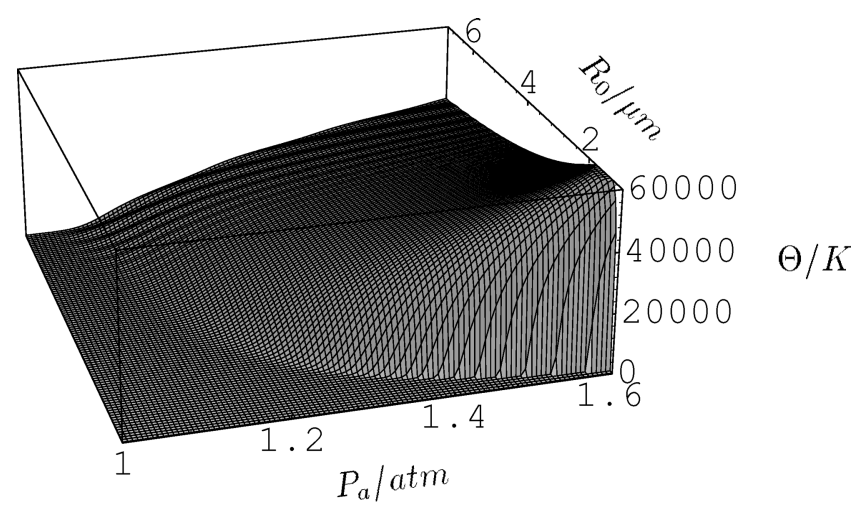

FIG. 8. Maximal temperature in the bubble as a function of $R_{0}$ and $P_{a}$ within our model calculation. 
If $c^{\mathrm{Ar}}(r, t)$ and $c^{\mathrm{N}_{2}}(r, t)$ are the concentration fields of Ar and $\mathrm{N}_{2}$ in the liquid, respectively, the rate of change of the number of moles of $\mathrm{N}_{2}$ and $\mathrm{Ar}$ in the bubble is given by

$$
\begin{aligned}
& \dot{N}_{\mathrm{Ar}}=\frac{\left.4 \pi R^{2} D_{\mathrm{Ar}} \partial_{r} c^{\mathrm{Ar}}\right|_{r=R}}{\mu_{\mathrm{Ar}}}, \\
& \dot{N}_{\mathrm{N}_{2}}=\frac{4 \pi R^{2} D_{\mathrm{N}_{2}} \partial_{r} c^{\left.\mathrm{N}_{2}\right|_{r=R}}}{\mu_{\mathrm{N}_{2}}}-A N_{\mathrm{N}_{2}} \exp \left(-\frac{\Theta^{*}}{\Theta}\right) .
\end{aligned}
$$

Here, $D_{\mathrm{Ar}}, D_{\mathrm{N}_{2}}, \mu_{\mathrm{Ar}}$ and $\mu_{\mathrm{N}_{2}}$ are the respective diffusion constants and molecular masses. The concentration fields obey a mass advection diffusion equation, ${ }^{18,20}$ whose boundary conditions are set by the external concentrations

$$
c^{\alpha}(\infty, t)=c_{\infty}^{\alpha}=c_{0}^{\alpha} \frac{p_{\infty}^{\alpha}}{P_{0}}
$$

(Henry's law) and by the partial gas pressures $p^{\alpha}(t)$ in the bubble

$$
c^{\alpha}(R(t), t)=c_{0}^{\alpha} \frac{p^{\alpha}(R(t))}{P_{0}},
$$

$\alpha=\operatorname{Ar}, \mathrm{N}_{2}$. The solubilities for nitrogen and argon are different, $c_{0}^{\mathrm{Ar}}=0.061 \mathrm{~kg} / \mathrm{m}^{3}$ and $c_{0}^{\mathrm{N}_{2}}=0.020 \mathrm{~kg} / \mathrm{m}^{3} .{ }^{52}$ The diffusion constants are approximately the same, ${ }^{53} D_{\mathrm{Ar}}=D_{\mathrm{N}_{2}}$ $=2 \times 10^{-9} \mathrm{~m}^{2} / \mathrm{s}$. The second term in Eq. (12) represents the bubble's nitrogen loss by chemical reaction. The reaction rate will depend on the temperature $\Theta(t)$ in the bubble. For simplicity, we assume that the reactions follow an Arrhenius law, with empirical parameters appropriate for nitrogen dissociation (Ref. 51): $A=6 \times 10^{19}\left(\Theta_{0} / \Theta\right)^{2.5}\left(\rho_{0} / \mu_{\mathrm{N}_{2}}\right)$ $\times\left(R_{0} / R\right)^{3} \mathrm{~cm}^{3} /(\mathrm{mol} \mathrm{s})$ giving the time scale of the reaction; $\Theta^{*}=113000 \mathrm{~K}^{51}$ is the activation temperature and $\rho_{0}$ the equilibrium gas density. This reaction law is rather crude, as it neglects backward reactions as well as the kinetics of the expulsion of reaction products; however, it is sufficient for this demonstrative calculation.

We can straightforwardly extend the adiabatic approximation of the slow diffusional dynamics ${ }^{15,18,20}$ (i.e., separation of times scales) to Equations (11), (12). The only requirement is that the involved chemical reactions are fast compared to diffusional processes which definitely is the case. The result of the adiabatic approximation is that the change per cycle is given by

$$
\begin{aligned}
\frac{\Delta N_{A r}}{T}= & \frac{4 \pi D_{\mathrm{Ar}} c_{0}^{\mathrm{Ar}}}{\mu_{\mathrm{Ar}} P_{0} I}\left(p_{\infty}^{\mathrm{Ar}}-\xi_{b}\langle p\rangle_{4}\right), \\
\frac{\Delta N_{\mathrm{N}_{2}}}{T}= & \frac{4 \pi D_{\mathrm{N}_{2}} c_{0}^{\mathrm{N}_{2}}}{\mu_{\mathrm{N}_{2}} P_{0} I}\left(p_{\infty}^{\mathrm{N}_{2}}-\left(1-\xi_{b}\right)\langle p\rangle_{4}\right) \\
& -N_{\mathrm{N}_{2}}\langle A \exp (-\Theta * / \Theta)\rangle_{0}
\end{aligned}
$$

with the weighted time averages

$$
\langle f(t)\rangle_{i}=\frac{\int_{0}^{T} f(t) R^{i}(t) d t}{\int_{0}^{T} R^{i}(t) d t} .
$$

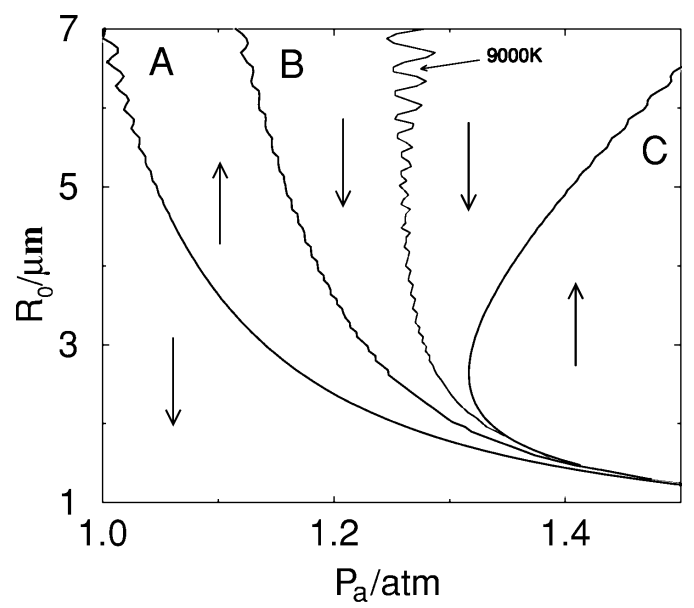

FIG. 9. Phase diagram for air at $p_{\infty} / P_{0}=0.20$ in the $R_{0}-P_{a}$ space. The arrows denote whether the ambient radius grows or shrinks at this parameter value. Curve $A$ denotes the equilibrium for an air bubble, on curve $C$ the bubble only contains argon. The intermediate curve B necessarily exists because of the topology of the diagram, and represents an additional stable equilibrium. The thin line shows when the nitrogen dissociation threshold $9000 \mathrm{~K}$ is reached.

For further simplification in the numerical calculations to come we in addition employ the saddle point approximation of the integral $I$ defined by

$$
I=\int_{0}^{\infty} \frac{d h^{\prime}}{\left\langle\left(3 h^{\prime}+R^{3}(t)\right)^{4 / 3}\right\rangle_{0}}
$$

as $I \approx 1 / R_{\max }$, as already done in Ref. 15 .

The last term in Eq. (14) will only contribute at the collapse when $\Theta(t)$ is large. The chemical reaction rate will depend on the detailed space and time dependence of the temperature in the bubble which we model by the polytropic law (7)-as explained in the previous section.

With these approximations equilibrium points $\Delta N_{\mathrm{Ar}}=\Delta N_{\mathrm{N}_{2}}=0$ in the two dimensional space $\left(N_{\mathrm{Ar}}, N_{\mathrm{N}_{2}}\right)$, or equivalently in the space $\left(\xi_{b}, R_{0}\right)$, can easily be calculated from the RP dynamics (4) via Equations (13), (14). As in Ref. 18 , only averages of type (15) have to be determined for each parameter pair $\left(R_{0}, P_{a}\right)$; therefore, phase diagrams can be calculated solely from the Rayleigh-Plesset equation (4). The equilibrium radii $R_{0}^{*}$ in the $R_{0}-P_{a}$ plane for air $\left(\xi_{l}=0.01\right)$ at $p_{\infty} / P_{0}=0.20$ are shown in Figure 9 . For small forcing the temperatures are not high enough to initiate chemical reactions, so that the equilibrium curve corresponds to the prediction of Ref. 18 for this gas concentration. This equilibrium is unstable: The bubble either shrinks or grows by rectified diffusion. As pointed out above the growing bubble eventually runs into a shape instability where microbubbles pinch off and make the bubble dance because of the recoil. ${ }^{18}$ In the opposite limit of high forcing (curve $\mathrm{C}$ ), the reactions burn off all the $\mathrm{N}_{2}$, so that the bubble contains pure argon; this equilibrium corresponds to the (stable) equilibrium at the argon partial pressure $p_{\infty}^{\mathrm{Ar}} / P_{0}=0.01 p_{\infty} /$ $P_{0}=0.002$.

Figure 9 displays a regime of shrinking bubbles at high forcing pressures (left of curve $\mathrm{C}$ ) and an adjacent region of growing bubbles (right of curve A). This necessitates the 


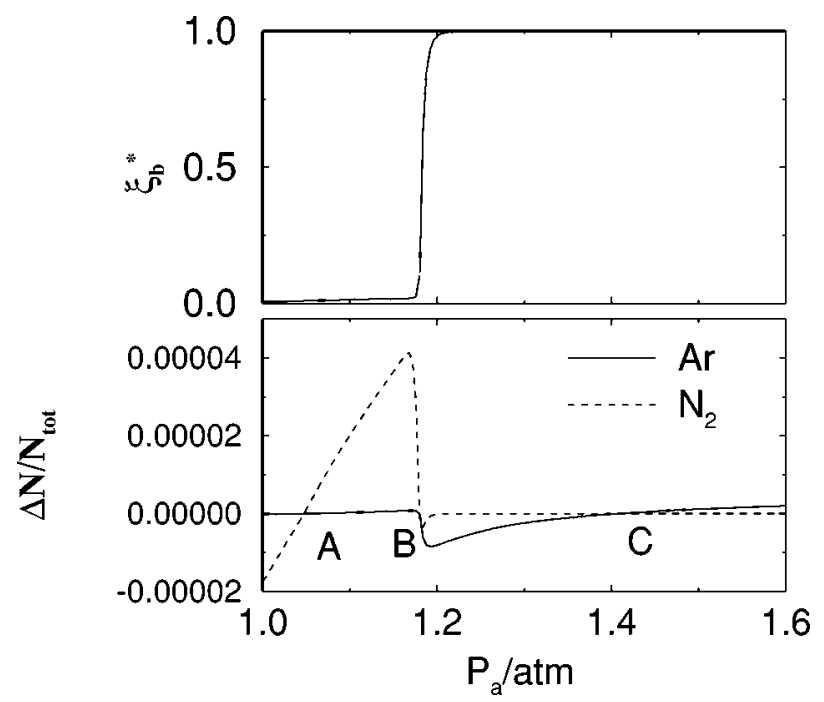

FIG. 10. The top curve shows the equilibrium argon ratio $\xi_{b}^{*}$ in a $R_{0}=5 \mu \mathrm{m}$ bubble as a function of the forcing pressure $P_{a}$. The bottom curve presents the corresponding argon and nitrogen loss/gain per cycle, normalized to the total amount of moles.

existence of an additional equilibrium at intermediate forcing pressures, curve B in Figure 9, for which growth by rectified diffusion and mass loss by reactions balance. This additional equilibrium occurs close to the point of nitrogen dissociation, and turns out to be stable; the argon fraction $\xi_{b}^{*}$ for this equilibrium is slightly larger than the fraction $\xi_{l}$ in the liquid (for not too strong forcing).

In Figure 10 we present the net loss/gain per cycle for both argon and nitrogen for a $R_{0}=5 \mu \mathrm{m}$ bubble. At small $P_{a}<1.15$ atm the second term in Equation (14) is not important as the gas does not become hot enough. The bubble diffusively shrinks (for $P_{a}<1.045$ atm) or grows because of rectified diffusion; the argon ratio is constant at $\xi_{b}^{*}=\xi_{l}=0.01$. In between the shrinking and the growing regime is the unstable equilibrium point A. Around $P_{a}=1.17 \mathrm{~atm}$ the heating is sufficient to lead to some nitrogen dissociation and at $P_{a}=1.18 \mathrm{~atm}$ the nitrogen loss through dissociation balances its gain through rectified diffusion. At that forcing pressure also argon is in equilibrium as the argon ratio has increased up to $\xi_{b}^{*} \approx 0.1$ so that $p_{\infty}^{\mathrm{Ar}}=\xi_{b}^{*}\langle p\rangle_{4}$ : We have reached the stable equilibrium point B. At larger forcing pressure $P_{a}$ the bubble is in the dissolution island. For increasing $P_{a}$ the nitrogen loss becomes less because less and less nitrogen is left in the bubble. The argon loss, on the other hand, diminishes because we approach the stable diffusive equilibrium point $\mathrm{C}$ at $P_{a} \approx 1.4 \mathrm{~atm}$. Beyond this forcing pressure, the bubble grows again by rectified argon diffusion; essentially no nitrogen is left in the bubble $\left(1-\xi_{b}^{*} \approx 6 \times 10^{-5}\right.$ at $\left.P_{a}=1.40 \mathrm{~atm}\right)$. Note that because of the separation of time scales the values on $\xi_{b}^{*}$ refer to an average value over the whole cycle. When the bubble is close to its maximum, the nitrogen concentration will be slightly larger, right at the collapse the argon concentration will be slightly larger.

How robust is this picture, i.e., how does it depend on

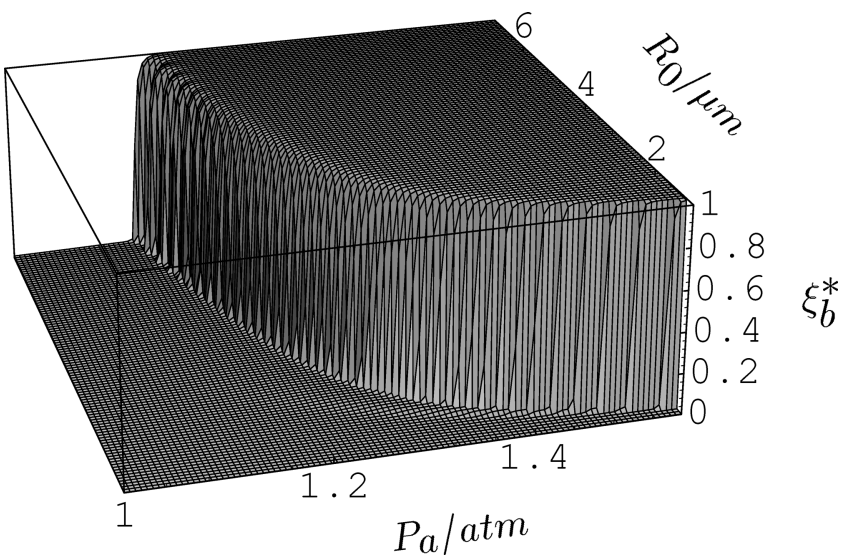

FIG. 11. The fraction $\xi_{b}^{*}$ of argon in the bubble as a function of $P_{a}$ and $R_{0}$.

details of temperature dynamics and the chemical reaction mechanism which are both poorly understood? The large $R_{0}$ parts of branches $\mathrm{A}$ and $\mathrm{C}$ do not depend at all on these details. The reason becomes clear from Figure 11 in which we show the equilibrium composition $\xi_{b}^{*}$ as a function of $R_{0}$ and $P_{a}$. It is given by

$$
\frac{\Delta N_{\mathrm{Ar}}\left(\xi_{b}^{*}\right)}{\Delta N_{\mathrm{N}_{2}}\left(\xi_{b}^{*}\right)}=\frac{\xi_{b}^{*}}{1-\xi_{b}^{*}} \text {. }
$$

Plugging (13) and (14) into (17) gives the quadratic equation for the equilibrium argon ratio $\xi_{b}^{*}$,

$$
(\alpha+\epsilon) \xi_{b}^{* 2}+(\beta-\epsilon) \xi_{b}^{*}+\delta=0
$$

with the coefficients

$$
\begin{aligned}
& \alpha=\frac{4 \pi}{I} \frac{\langle p\rangle_{4}}{P_{0}}\left(\frac{D_{\mathrm{N}_{2}} c_{0}^{\mathrm{N}_{2}}}{\mu_{\mathrm{N}_{2}}}-\frac{D_{\mathrm{Ar}} c_{0}^{\mathrm{Ar}}}{\mu_{\mathrm{Ar}}}\right), \\
& \beta=\frac{4 \pi}{I P_{0}}\left(\frac{D_{\mathrm{N}_{2}} c_{0}^{\mathrm{N}_{2}}}{\mu_{\mathrm{N}_{2}}}\left(p_{\infty}^{\mathrm{N}_{2}}-\langle p\rangle_{4}\right)+\frac{D_{\mathrm{Ar}} c_{0}^{\mathrm{Ar}}}{\mu_{\mathrm{Ar}}}\left(p_{\infty}^{\mathrm{Ar}}+\langle p\rangle_{4}\right)\right), \\
& \epsilon=\frac{4 \pi P_{0} R_{0}^{3}}{3 G \Theta_{0}}\left\langle A \exp \left(-\frac{\Theta^{*}}{\Theta}\right)\right\rangle_{0}, \\
& \delta=-\frac{4 \pi D_{\mathrm{Ar}} c_{\infty}^{\mathrm{Ar}}}{I \mu_{\mathrm{Ar}}} .
\end{aligned}
$$

From the two solutions only the one with $0 \leqslant \xi_{b}^{*} \leqslant 1$ has physical meaning. The interpretation of Figure 11 is straightforward: Weakly forced bubbles have $\xi_{b}^{*} \approx \xi_{l}$, thus $p_{\infty} / P_{0}=0.20$ is relevant for stability. Strongly forced bubbles have $\xi_{b}^{*} \approx 1 \gg \xi_{l}$, thus $p_{\infty}^{\mathrm{Ar}} / P_{0}=0.002$ is the relevant quantity. The transition between these regimes is abrupt, and occurs when the bubble temperature surpasses the dissociation temperature $\left(\approx 9000 \mathrm{~K}\right.$ for $\left.\mathrm{N}_{2}\right)$. Where exactly this happens depends on the model of the temperature and thus so does the equilibrium curve B in Figure 9.

However, the existence of this additional stable equilibrium is independent of the model details. It simply follows from topological reasons: To the right of the curve A the 


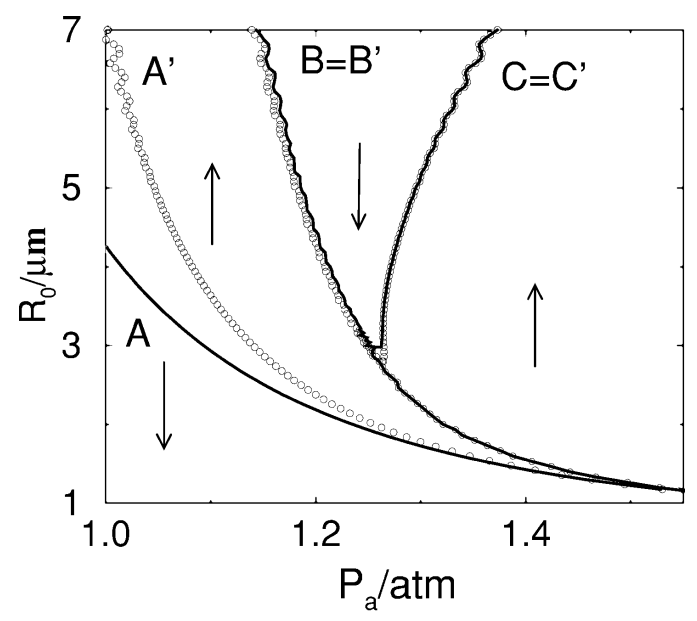

FIG. 12. The solid lines (curves A, B, C) show the same phase diagram for air as in Figure 9, but now for $p_{\infty} / P_{0}=0.50$, i.e., $p_{\infty}^{\mathrm{Ar}} / P_{0}=0.005$. For comparison, the open symbols (curves $\mathrm{A}^{\prime}, \mathrm{B}^{\prime}, \mathrm{C}^{\prime}$ ) show the phase diagram for a nitrogen-argon mixture with an argon ratio of $\xi_{l}=0.025$ at $p_{\infty} / P_{0}=0.20$, which beyond the nitrogen dissociation threshold results in the same partial argon pressure $p_{\infty}^{\mathrm{Ar}} / P_{0}=0.005$. Indeed, for large $P_{a}$ the phase diagrams agree. Only curves $\mathrm{A}$ and $\mathrm{A}^{\prime}$ below the nitrogen dissociation threshold differ.

bubbles are growing, to the left of curve $\mathrm{C}$ they are shrinking, so in between there must be an equilibrium. Indeed, as mentioned already above and shown in Figure 5, Holt and Gaitan's ${ }^{22}$ recent detailed measurements of $R_{0}-P_{a}$ phase diagrams found such a classically unexpected equilibrium (for the same air pressure $p_{\infty} / P_{0}=0.20$ as chosen here) and the connected large $P_{a}$ " dissolution island" between curves $\mathrm{B}$ and $\mathrm{C}$.

Holt and Gaitan ${ }^{22}$ repeated the experimental phase space measurements for larger air pressures $p_{\infty} / P_{0}=0.40$ and $p_{\infty} / P_{0}=0.50$. The theoretical phase diagram for $p_{\infty} / P_{0}=0.50$ and $\xi_{l}=0.01$ (air) is shown in Figure 12. The island of dissolution between curves $\mathrm{B}$ and $\mathrm{C}$ is now smaller, as the partial argon pressure is $p_{\infty}^{\mathrm{Ar}} / P_{0}=0.005$ and curve $\mathrm{C}$ is thus shifted to the left, whereas the nitrogen dissociation curve is hardly affected and consequently neither are curves $\mathrm{A}$ and $\mathrm{B}$. In experiment we see the same qualitative behavior, but even more strongly pronounced, see Figure 1a of Ref. 22. We expect the poor modeling of both the temperature dependence and the chemistry as the origin of the quantitative disagreement of the theoretical and experimental phase diagrams.

For comparison we also calculated the phase diagram for $p_{\infty} / P_{0}=0.20$ and $\xi_{l}=0.025$ and plotted it into the same Figure 12. For this situation the argon percentage $p_{\infty}^{\mathrm{Ar}} / P_{0}=\xi_{l} p_{\infty} / P_{0}=0.005$ is the same as for $p_{\infty} / P_{0}=0.50$, $\xi_{l}=0.01$. Indeed, the phase diagram demonstrates very convincingly that for large forcing pressure it is only the partial argon pressure which is the relevant parameter for diffusive stability: The equilibrium curves $\mathrm{B}$ and $\mathrm{C}$ for the two cases agree very well.

\section{PHASE DIAGRAMS FOR FURTHER NITROGEN- ARGON MIXTURES}

Our theoretical method can be applied to many other experimental situations. As a further example we calculate

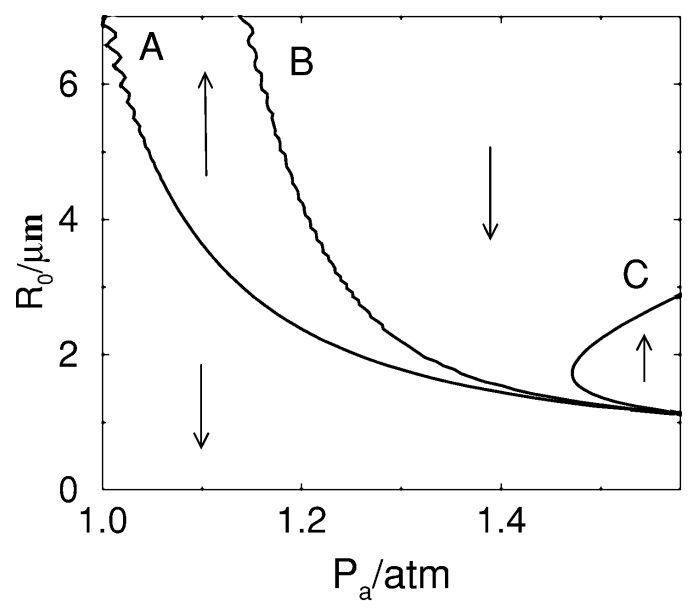

FIG. 13. $R_{0}-P_{a}$ phase diagram for nitrogen with a small amount $\xi_{l}=0.001$ of argon dissolved. The relative pressure overhead is $p_{\infty} / P_{0}=0.20$. These parameters correspond to the experimental situation in Figure 11 of Löfstedt et al. (Ref. 15), see Fig. 4 of the present work.

the corresponding phase diagram Figure 13 to the experimental situation in Figure 11 of Ref. 15, see Figure 4 here, where $p_{\infty} / P_{0}=0.20$ and $\xi_{l}=0.001$. The total gas concentration $p_{\infty} / P_{0}$ has the same value as in Figure 9 . Therefore, the curves A and B are also essentially as in that figure, because they are determined by the nitrogen concentration. Curve $\mathrm{C}$ which is beyond the nitrogen dissociation threshold, however, is further to the right, reflecting the lower argon concentration $p_{\infty}^{\mathrm{Ar}} / P_{0}=0.0002$.

What happens if $P_{a}$ is slowly increased? For small $P_{a}=1 \mathrm{~atm}$ the bubble will be repelled from the unstable equilibrium A. It either dissolves or grows by rectified diffusion and runs into the shape instability where it pinches off a microbubble and the whole process starts over, as described in detail in Ref. 18. For $P_{a} \approx 1.15$ atm the bubble does not run into the shape instability any more but in the attractive diffusive equilibrium curve B where it stays. On further increase of $P_{a}$ it follows that curve and shrinks drastically. Exactly this sequence of events has experimentally been observed, see Figure 11 of Löfstedt et al. ${ }^{15}$

For larger $P_{a}$ on first sight there seems to be a contradiction to the experimental observation Figure 11 of Ref. 15. Theoretically, the bubble should always stay on the stable equilibrium curve B. Experimentally, however, between $P_{a}=1.3 \mathrm{~atm}$ and $1.4 \mathrm{~atm}$ no bubble seems to exist and at $P_{a}=1.4 \mathrm{~atm}$ the ambient radius is much larger, similar to that of curve $C$ rather than that of curve $B$.

This variance may be resolved when we consider the unavoidable jitter in the forcing pressure amplitude $P_{a}$. At $P_{a}=1.35 \mathrm{~atm}$, the equilibrium curves $\mathrm{A}$ and $\mathrm{B}$ are so close that a small jitter $\Delta P_{a}$ (to smaller $P_{a}$ ) of less than $0.05 \mathrm{~atm}$ (which is less than the precision to which $P_{a}$ can be determined; also the translational movements of the bubble and its distortion on the pressure field ${ }^{54}$ in the liquid could contribute to a jitter) suffices to make the bubble jump to the unstable domain below curve A where it dissolves. Thus, though the equilibrium $\mathrm{B}$ in that regime is stable from a 

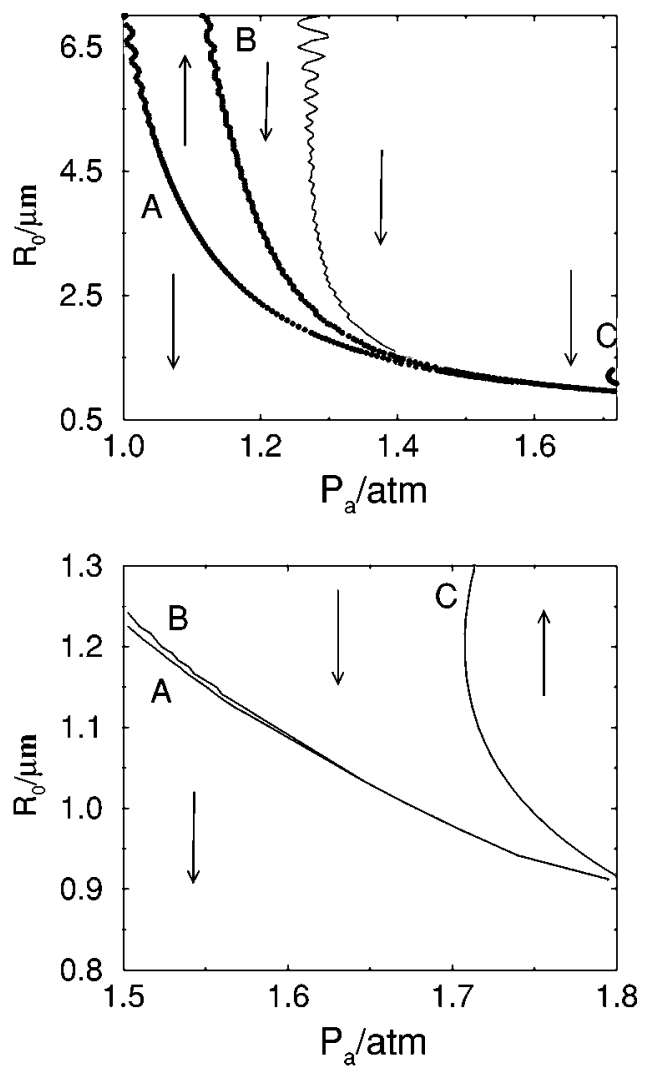

FIG. 14. Phase diagram in $R_{0}-P_{a}$ space for $p_{\infty} / P_{0}=0.20$ and a tiny argon concentration of $\xi_{l}=0.0001$. Only for very high forcing pressure diffusively stable bubbles are possible (but are likely to be unstable towards the surface shape instability). The bottom figure shows an enlargement of the lower right part of the top figure.

mathematical point of view, from a physical point of view it may be not and the bubble can dissolve. Support for this claim could come from an experiment in which the jitter is artificially increased: the gap in Figure 4 where no bubble exists should widen.

With the help of the phase diagram Figure 13 we now better understand the above-mentioned hysteresis. The stable part of branch $\mathrm{C}$ is reached by a fast increase of $P_{a}$. The bubble is then boosted into the growing regime below the stable branch of curve C. Subsequently, this stable branch C is reached through rectified diffusion. On the other hand, if $P_{a}$ is decreased from $P_{a}=1.4 \mathrm{~atm}$, the bubble will first follow the stable equilibrium curve $C$ and shrink. At the bifurcation point it falls off the equilibrium curve and shrinks toward the stable curve B. Because of the jitter in $P_{a}$ curve B may not be able to trap the bubble as explained above and the bubble dissolves. Even a fast decrease of $P_{a}$ toward much smaller values $\approx 1.1$ atm does not save the bubble from dissolution as it only contains argon and the nitrogen needed for a stable equilibrium has been burned off in the first part of the cycle during which $P_{a}$ was increased and recollecting nitrogen only happens on the slow diffusive time scale.

For even lower argon concentration as, e.g., in Figure 14 where we chose $\xi_{l}=0.0001$ and $p_{\infty} / P_{0}=0.20$ the situation is similar. The only difference is that curve $\mathrm{C}$ moves even fur- ther to the right and may not be reached in experiment because of the (short time scale) shape instabilities discussed in Refs. 18 and 25.

Let us discuss the situation in the limiting case $\xi_{l}=0$, i.e., pure nitrogen. As explained above for $\xi_{l}=0.001$, on increasing $P_{a}$ the bubble should first "dance" because of growth by rectified diffusion and pinching off of microbubbles and then it should be caught by the stable (but not necessarily sonoluminescing) equilibrium curve B. At this equilibrium bubble growth by rectified diffusion and nitrogen loss by dissociation again just balance. For large $P_{a} \sim 1.3 \mathrm{~atm}$ the bubble should dissolve by the same mechanism discussed above: Curves A and B are so close that a jitter in $P_{a}$ leads to a jump from the stable equilibrium curve $\mathrm{B}$ to the dissolution domain below the unstable curve $\mathrm{A}$. We suggest to experimentally look for the stable equilibrium curve $\mathrm{B}$ which is suggested by the nitrogen dissociation hypothesis.

What experiments on pure nitrogen bubbles have been done up to now? To our knowledge only one experiment has been reported (Hiller et al. ${ }^{11}$ ). In that experiment the nitrogen was $99.7 \%$ pure. Hiller et al. ${ }^{11}$ managed to measure extremely weakly sonoluminescing unstable bubbles which were hard to keep alive. This supports the presented theory that in the strong forcing regime the high temperature destroys the nitrogen and thus the bubbles. However, the light intensity Figure 5 of Ref. 11 shows an oscillating pattern (on a time scale of seconds) which we do not understand.

\section{INERT GAS MIXTURES}

It is interesting to note that the chemical instability of one component of the gas mixture is not a necessary requirement for the accumulation of one gas species in the bubble. A similar accumulation can be achieved for inert gas mixtures if the diffusion constants and the equilibrium concentrations of the two gases in the mixtures are different. As an example we consider a 1:1 mixture of helium and argon at $p_{\infty} / P_{0}=0.20$, i.e., $\xi_{l}=0.50$ for the argon ratio. The diffusion constants are $D_{\mathrm{Ar}}=2 \times 10^{-9} \mathrm{~m}^{2} / \mathrm{s}$ and $D_{\mathrm{He}}=5.8 \times 10^{-9} \mathrm{~m}^{2} / \mathrm{s}^{53}$ and the equilibrium concentrations are $c_{0}^{\mathrm{Ar}}=61 \mathrm{~g} / \mathrm{m}^{3}$ and $c_{0}^{\mathrm{He}}=0.78 \mathrm{~g} / \mathrm{m}^{3} .{ }^{52}$ The molecular masses are $\mu_{\mathrm{Ar}}=40 \mathrm{~g} / \mathrm{mol}$ and $\mu_{\mathrm{He}}=4 \mathrm{~g} / \mathrm{mol}$. The resulting equilibrium concentration $\xi_{b}^{*}$ in the bubble is shown in Figure 15. For small forcing, helium accumulates in the bubble and for large forcing, argon accumulates. The borderline between these two regimes is the unstable diffusive equilibrium curve calculated in Ref. 18 and denoted "curve A" within the plots shown here. As no reactions occur, this is the only equilibrium: Below that curve bubbles diffusively shrink, above it they grow by rectified diffusion. From this behavior the helium or argon accumulation can immediately be qualitatively understood: The diffusive change of mass is proportional to the product of the material constants $D c_{0} / \mu$, see Eq. (13). Now $D_{\mathrm{Ar}} c_{0}^{\mathrm{Ar}} / \mu_{\mathrm{Ar}}=3.1 \times 10^{-9} \mathrm{~mol} \mathrm{~s}^{-1} \mathrm{~m}^{-1}$ and $D_{\mathrm{He}} c_{0}^{\mathrm{He}} / \mu_{\mathrm{He}}=1.1$ $\times 10^{-9} \mathrm{~mol} \mathrm{~s}^{-1} \mathrm{~m}^{-1}$. Thus in the shrinking regime argon can escape faster and helium accumulates. By contrast, in the growing regime the rectified diffusion of argon into the 


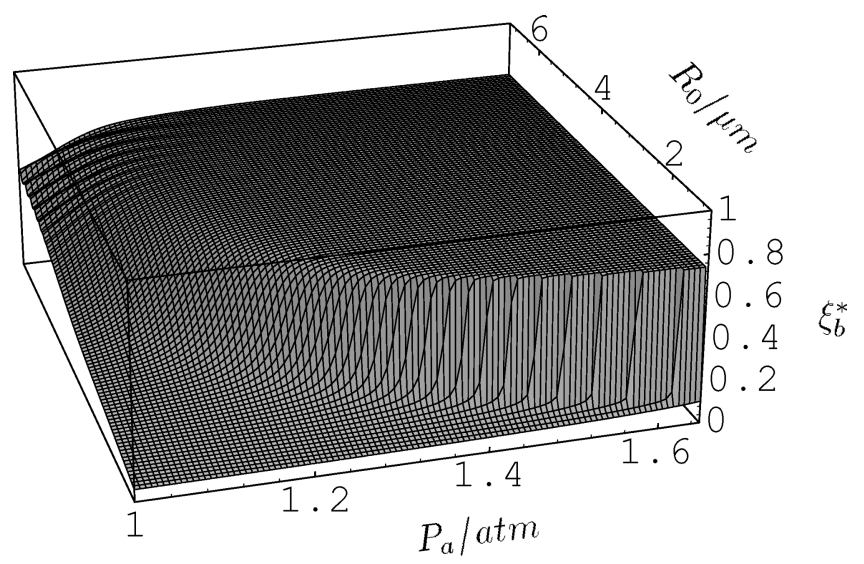

FIG. 15. Argon equilibrium ratio $\xi_{b}^{*}$ in a bubble as a function of ambient radius $R_{0}$ and forcing pressure $P_{a}$. The external gas is a 1:1 mixture of argon and helium at an overhead pressure of $p_{\infty} / P_{0}=0.20$. Only the difference in the diffusion constants and in the equilibrium concentrations between argon and helium leads to the argon accumulation in the bubble for large forcing.

bubble wins and it accumulates. In the process of helium accumulation the helium equilibrium concentration $1-\xi_{b}^{*}$ may never be achieved as the bubble dissolves earlier. This problem does not occur for the process of argon accumulation, as the diffusively growing bubble runs into the shape instability and can survive after microbubble pinchoff. ${ }^{18}$

The maximal argon concentration achieved is only $\xi_{b, \max }^{*} \approx 0.73$, less than the result $\xi_{b, \max }^{*}=1$ of the previous section where the chemical instability of nitrogen caused its complete extinction. Also the transition in Figure 15 is less abrupt than in the above case Figure 11 for which chemical reactions occurred.

The maximal argon concentration can easily be calculated analytically from equations (18)-(22) with the index $\mathrm{N}_{2}$ replaced by the index He. Obviously, $\epsilon=0$ as no reactions take place. From Figure 7 of Ref. 18 we realize that for large forcing $\langle p\rangle_{4}$ is small compared to $p_{\infty}^{\mathrm{Ar}}=p_{\infty}^{\mathrm{He}}=0.1$. With this large forcing pressure approximation we obtain

$$
\xi_{b, \text { max }}^{*}=\frac{\frac{D_{\mathrm{Ar}} c_{\infty}^{\mathrm{Ar}}}{\mu_{\mathrm{Ar}}}}{\frac{D_{\mathrm{Ar}} c_{\infty}^{\mathrm{Ar}}}{\mu_{\mathrm{Ar}}}+\frac{D_{\mathrm{He}} c_{\infty}^{\mathrm{He}}}{\mu_{\mathrm{He}}}} \approx 0.73
$$

in agreement with the numerical result Figure 15.

\section{CONCLUSION, PREDICTIONS, AND SUGGESTED EXPERIMENTS}

We conclude this paper with a summary of the nitrogen dissociation theory, ${ }^{28,29}$ which we elaborated here in detail. It accounts for the dependence of SBSL on the percentage of inert gas in the bubble. It is based on a combination of principles from sonochemistry and hydrodynamic stability. The main result is that strongly forced air bubbles eventually consist of pure argon because at the high temperatures achieved in the bubble the nitrogen and oxygen molecules dissociate and react to water soluble gases. Consequently, it is the par- tial pressure $p_{\infty}^{\mathrm{Ar}}$ of argon (or of any other inert gas) which is relevant for stability. ${ }^{29}$ The dynamics of the bubble is described by the Rayleigh-Plesset equation; chemical reactions are assumed to obey an Arrhenius type law; back reactions are neglected. The bubble pressure is approximated by a van der Waals law. The slow diffusive processes can be treated in an adiabatic approximation. The theory contains no adjustable fit parameter.

Finally, we make detailed suggestions of experiments to further test the nitrogen dissociation hypothesis.

(1) Phase diagrams in the $p_{\infty} / P_{0}$ vs $P_{a} / P_{0}$ parameter space: The most basic prediction is that in the large forcing regime the partial pressure $p_{\infty}^{\mathrm{Ar}} / P_{0}$ is the relevant parameter for obtaining stable SL (or the partial pressure of any other inert gas). We suggest mapping out the phase diagram in Figure 2 for argon-nitrogen mixtures at various pressure overheads $p_{\infty} / P_{0}$ and various argon ratios $\xi_{l}$ : (i) Only the partial argon pressure $p_{\infty}^{\mathrm{Ar}} / P_{0}=\xi_{l} p_{\infty} / P_{0}$ should matter and (ii) the measured phase diagram should look like the theoretical one in Figure 2.

(2) Stable SL without degassing: In particular, stable SL will be possible without degassing, i.e., at $p_{\infty} / P_{0}=1$, if the argon ratio is properly adjusted. For $P_{a}=1.3 \mathrm{~atm}$ it must be $\xi_{l} \approx 0.0033$. To test this prediction, extremely pure water has to be used to avoid spontaneous cavitation. An alternate way to achieve stable SL without degassing is to increase the ambient pressure correspondingly.

(3) Oxygen-argon mixtures: Oxygen has a dissociation temperature which is only slightly lower than that of nitrogen. Therefore, oxygen-argon mixtures should behave very similar to nitrogen-argon mixtures. In particular, at large forcing only argon is left in the bubble, so even the light intensities and spectra should be very similar. This prediction has recently been confirmed in experiment. ${ }^{55}$

(4) Other inert gas doped molecular gases: Apart from $\mathrm{CO}$, nitrogen is the most stable molecular gas. Other molecular gases dissociate earlier. Therefore, mixtures of them with inert gases will show a slight shift of the stable equilibrium curve B toward smaller forcing pressure. As the temperature increase with forcing pressure is very sudden (see Figure 8), this shift will, however, be very small and may be undetectable.

(5) Stable nitrogen bubbles: Another very basic prediction of the nitrogen dissociation theory is the existence of the equilibrium curve $\mathrm{B}$ in the phase diagrams of the type shown in Figure 9. For air Holt and Gaitan have observed it. ${ }^{22}$ We predict that it exists for any reactive gas. In particular, we expect that pure nitrogen bubbles can be stable.

(6) Island of bubble dissolution for large $P_{a}$ : Between the equilibrium curves $\mathrm{B}$ and $\mathrm{C}$, bubbles dissolve. The size of this large $P_{a}$ island of dissolution should only depend on the partial argon pressure $p_{\infty}^{\mathrm{Ar}} / P_{0}=\xi_{l} p_{\infty} / P_{0}$, hardly on the argon ratio $\xi_{l}$ or the pressure overhead $p_{\infty} / P_{0}$ individually. For larger $p_{\infty}^{\mathrm{Ar}} / P_{0}$ the size of the dissolution regime should shrink, for smaller $p_{\infty}^{\mathrm{Ar}} / P_{0}$ it should grow.

(7) Radon doped SL bubbles: By using radon-nitrogen gas mixtures it may be possible to detect the inert gas accu- 
mulation in the bubble because of the radioactivity of radon.

(8) Inert gas accumulation: Even for a mixture of nonreactive gases as $\mathrm{He}$ and $\mathrm{Ar}$ we in general have $\xi_{b} \neq \xi_{l}$. In the large forcing pressure regime argon accumulates for this example.

(9) $\mathrm{pH}$ measurements for air bubbles: A very appealing test is to measure the concentration of the reaction products of the dissociated gases as a function of time, as already done in MBSL. ${ }^{38}$ For nitrogen-argon mixtures nitrous acid production would lead to a decrease in $\mathrm{pH}$ as already predicted in Ref. 29. For an estimate of the production rate we assume that all the nitrogen that diffuses into the bubble during the bubble expansion is burned off at the collapse. This amount is estimated in Ref. 15 as

$$
\Delta N_{\mathrm{N}_{2}}=\frac{2 \pi D_{\mathrm{N}_{2}} c_{\infty}^{\mathrm{N}_{2}} R_{\max } T}{\mu_{\mathrm{N}_{2}}}
$$

per cycle. With typical values of $R_{\max }=10 R_{0}$ for the maximal radius, $R_{0}=5 \mu \mathrm{m}, D_{\mathrm{N}_{2}}=2 \times 10^{-9} \mathrm{~m}^{2} / \mathrm{s}, c_{\infty}^{\mathrm{N}_{2}} \approx 0.20 c_{0}^{\mathrm{N}_{2}}$, $c_{0}^{\mathrm{N}_{2}}=0.02 \mathrm{~kg} / \mathrm{m}^{3}$, and $T=38 \mu \mathrm{s}$ one obtains $\Delta N_{\mathrm{N}_{2}} \approx 3 \times 10^{-18} \mathrm{~mol}$ per cycle or $\sim 3 \times 10^{-10} \mathrm{~mol}$ of $\mathrm{N}_{2}$ per hour converted to reaction products. The consequence is a small but detectable $\mathrm{pH}$ decrease: $\mathrm{In}$ a $100 \mathrm{ml}$ flask with pure water $(\mathrm{pH}=7)$ there are initially $10^{-8} \mathrm{~mol} \mathrm{H}^{+}$ions. Assume that all ejected $\mathrm{N}$ atoms eventually form either $\mathrm{HNO}_{2}$ or $\mathrm{HNO}_{3}$, then $6 \times 10^{-10} \mathrm{~mol} \mathrm{H}^{+}$ions are produced. This means a concentration increase to $\left(10^{-7}+6 \times 10^{-9}\right) \mathrm{mol} / \mathrm{l}$ or a $\mathrm{pH}$ value of 6.975 after an hour.

(10) pH measurements for bubbles of other gas mixtures: As a blind test one should also measure the $\mathrm{pH}$ as a function of time for pure argon bubbles. No $\mathrm{pH}$ change should occur. Neither do we expect a pH change for oxygenargon mixtures as the main reaction product of the dissociated oxygen should be $\mathrm{H}_{2} \mathrm{O}_{2}$.

\section{ACKNOWLEDGMENTS}

We are grateful to Michael Brenner for our joint development of the Rayleigh-Plesset SL bubble approach. This ongoing collaboration has always been a pleasure to all of us. We thank him for lots of discussions and exchange over the last years. Without him this work would not have been possible. Many thanks also go to Todd Dupont, Siegfried Grossmann, Leo Kadanoff, Blaine Johnston, David Oxtoby, and Ken Suslick. We would also like to acknowledge Brad Barber's remark that high partial pressure air bubbles experimentally behave similar to low partial pressure argon bubbles, see Ref. 13. Support for this work by the Deutsche Forschungsgemeinschaft (DFG) under Grant No. SBF185-D8 is acknowledged.

${ }^{1}$ H. Frenzel and H. Schultes, Z. Phys. Chem. 27B, 421 (1934).

${ }^{2}$ E. N. Harvey, J. Am. Chem. Soc. 61, 2392 (1939).

${ }^{3}$ H. Kuttruff, Acustica 12, 230 (1962).

${ }^{4}$ M. A. Margulis, Ultrasonics 23, 157 (1985).

${ }^{5}$ C. E. Brennen, Cavitation and Bubble Dynamics (Oxford University Press, Oxford, 1995).

${ }^{6}$ D. F. Gaitan, Ph.D. thesis, The University of Mississippi, 1990; D. F.
Gaitan, L. A. Crum, R. A. Roy, and C. C. Church, J. Acoust. Soc. Am. 91, 3166 (1992).

${ }^{7}$ L. A. Crum, Phys. Today 47, 22 (1994); S. J. Putterman, Sci. Am. 272, 32 (1995).

${ }^{8}$ B. P. Barber and S. J. Putterman, Nature (London) 352, 318 (1991).

${ }^{9}$ B. P. Barber and S. J. Putterman, Phys. Rev. Lett. 69, 3839 (1992).

${ }^{10}$ R. Hiller, S. J. Putterman, and B. P. Barber, Phys. Rev. Lett. 69, 1182 (1992).

${ }^{11}$ R. Hiller, K. Weninger, S. J. Putterman, and B. P. Barber, Science 266, 248 (1994).

${ }^{12}$ R. Hiller and S. J. Putterman, Phys. Rev. Lett. 75, 3549 (1995), also see the erratum, Phys. Rev. Lett. 77, 2345 (1996).

${ }^{13}$ B. P. Barber, K. Weninger, R. Löfstedt, and S. J. Putterman, Phys. Rev. Lett. 74, 5276 (1995).

${ }^{14}$ R. Löfstedt, B. P. Barber, and S. J. Putterman, Phys. Fluids A 5, 2911 (1993).

${ }^{15}$ R. Löfstedt, K. Weninger, S. J. Putterman, and B. P. Barber, Phys. Rev. E 51, 4400 (1995)

${ }^{16}$ K. Weninger et al., J. Phys. Chem. 99, 14195 (1995).

${ }^{17}$ L. A. Crum, J. Acoust. Soc. Am. 57, 1363 (1975).

${ }^{18}$ S. Hilgenfeldt, D. Lohse, and M. P. Brenner, Phys. Fluids 8, 2808 (1996).

${ }^{19}$ A. Eller, J. Acoust. Soc. Am. 46, 1246 (1969); A. Eller and L.A. Crum, J. Acoust. Soc. Am. Suppl. 47, 762 (1970).

${ }^{20}$ M. M. Fyrillas and A. J. Szeri, J. Fluid Mech. 277, 381 (1994).

${ }^{21}$ M. P. Brenner, D. Lohse, D. Oxtoby, and T. F. Dupont, Phys. Rev. Lett. 76, 1158 (1996).

${ }^{22}$ G. Holt and F. Gaitan, Phys. Rev. Lett. 77, 3791 (1996).

${ }^{23}$ M. Plesset, J. Appl. Phys. 25, 96 (1954); H. W. Strube, Acustica 25, 289 (1971).

${ }^{24}$ A. Prosperetti, Q. Appl. Math. 34, 339 (1977).

${ }^{25}$ M. P. Brenner, D. Lohse, and T. F. Dupont, Phys. Rev. Lett. 75, 954 (1995).

${ }^{26}$ J. Holzfuss (private communication, 1997). Similar experiments were also made by F. Gaitan and G. Holt. The features of unstable SBSL are also seen in the acoustic signal, see J. Holzfuss, M. Rüggeberg, and A. Billo, Fortschritte der Akustik-DAGA 97, Bad Honnef: DPG GmbH (1997)

${ }^{27}$ R. G. Holt, D. F. Gaitan, A. A. Atchley, and J. Holzfuss, Phys. Rev. Lett. 72, 1376 (1994)

${ }^{28}$ M. P. Brenner, S. Hilgenfeldt, and D. Lohse, in Nonlinear Physics of Complex Systems-Current Status and Future Trends, edited by J. Parisi, S. C. Müller, and W. Zimmermann (Springer, Berlin, 1996), p. 79.

${ }^{29}$ D. Lohse, M. Brenner, T. Dupont, S. Hilgenfeldt, and B. Johnston, Phys. Rev. Lett. 78, 1359 (1997).

${ }^{30}$ A. Weissler, J. Acoust. Soc. Am. 25, 651 (1953).

${ }^{31}$ R. E. Verral and C. M. Sehgal, in Sonoluminescence in Ultrasound: Its Chemical, Physical and Biological Effects, edited by K. Suslick (VCH, Weinheim, 1988), p. 227.

${ }^{32}$ K. Suslick, Science 247, 1439 (1990); E. B. Flint and K. Suslick, ibid. 253, 1397 (1991); K. Suslick, E. B. Flint, M. W. Grinstaff, and K. A. Kemper, J. Phys. Chem. 97, 3098 (1993); K. Suslick, The Yearbook of Science and the Future 1994, Encyclopedia Britannica, Chicago, pp. 138155; T. Matula et al., Phys. Rev. Lett. 75, 2602 (1995).

${ }^{33} \mathrm{H}$. Schultes and H. Gohr, Angew. Chem. 49, 420 (1936).

${ }^{34}$ This value follows from the tabulated values for the enthalpy and the entropy changes, see, e.g., G. M. Barrow, Physical Chemistry (McGrawHill, New York, 1973). It is the value for which the Gibbs free energy $\Delta G$ passes through zero.

${ }^{35}$ L. Bernstein and M. Zakin, J. Phys. Chem. 99, 14619 (1995).

${ }^{36}$ B. P. Barber et al., Phys. Rev. Lett. 72, 1380 (1994).

${ }^{37}$ L. Kondic (private communication 1996); L. Kondic, C. Yuan, and C. K. Chan (preprint).

${ }^{38}$ E. Hart, Ch. H. Fischer, and A. Henglein, J. Phys. Chem. 91, 4166 (1987).

${ }^{39}$ M. P. Brenner, S. Hilgenfeldt, D. Lohse, and R. Rosales, Phys. Rev. Lett. 77, 3467 (1996)

${ }^{40}$ Lord Rayleigh, Philos. Mag. 34, 94 (1917); M. Plesset, J. Appl. Mech. 16, 277 (1949); A. Prosperetti, J. Acoust. Soc. Am. 56, 878 (1974); W. Lauterborn, ibid. 59, 283 (1976); A. Prosperetti, Ultrasonics 22, 69 (1984).

${ }^{41}$ A. Prosperetti, J. Acoust. Soc. Am. 61, 17 (1977).

${ }^{42}$ M. Plesset and A. Prosperetti, Annu. Rev. Fluid Mech. 9, 145 (1977)

${ }^{43}$ L. A. Crum, J. Acoust. Soc. Am. 73, 116 (1983).

${ }^{44}$ A. Prosperetti, L. A. Crum, and K. W. Commander, J. Acoust. Soc. Am. 83, 502 (1988).

${ }^{45}$ V. Q. Vuong and A. J. Szeri, Phys. Fluids 8, 2354 (1996). 
${ }^{46}$ V. Kamath, A. Prosperetti, and F. N. Egolfopoulos, J. Acoust. Soc. Am. 94, 248 (1993).

${ }^{47}$ K. Yasui, J. Acoust. Soc. Am. 98, 2772 (1995).

${ }^{48}$ Note that using unrealistic "effective" viscosities as large as seven times the real viscosity of water and also effective surface tensions as done by Putterman's group [(see Refs. 13, 14, and 36) and K. Weninger, B. Barber, and S. Putterman, Phys. Rev. Lett. 78, 1799 (1997)] is neither justified nor necessary to fit the data.

${ }^{49}$ C. C. Wu and P. H. Roberts, Phys. Rev. Lett. 70, 3424 (1993); Proc. R. Soc. London Ser. A 445, 323 (1994); W. Moss, D. Clarke, J. White, and D. Young, Phys. Fluids 6, 2979 (1994); Phys. Lett. A 211, 69 (1995); L. Kondic, J. Gersten, and C. Yuan, Phys. Rev. E 52, 4976 (1995); V. Q. Vuong and A. J. Szeri, Phys. Fluids 8, 2354 (1996).
${ }^{50}$ S. Hilgenfeldt, M. P. Brenner, S. Grossmann, and D. Lohse, J. Fluid Mech. (submitted).

${ }^{51}$ L. Bernstein, M. Zakin, E. Flint, and K. Suslick, J. Phys. Chem. 100, 6612 (1996).

${ }^{52}$ Handbook of Chemistry and Physics, edited by D. R. Lide (Chemical Rubber, Boca Raton, FL, 1991).

${ }^{53}$ Landolt and Börnstein, Zahlenwerte und Funktionen aus Physik und Chemie (Springer, Berlin, 1969).

${ }^{54}$ T. J. Matula, S. M. Cordry, R. A. Roy, and L. A. Crum, J. Acoust. Soc. Am. (submitted).

${ }^{55}$ D. Chow, K. Weninger, B. P. Barber, and S. J. Putterman, J. Acoust. Soc. Am. 100, 2718 (1996). 